\title{
Analytical Design of Multilayer Reflectors for Visible and Near IR Spectral Range by Contiguous Quarter-wave Stacks
}

\author{
M. Medhat, S. Y. El-Zaiat, M. F. Omar, S. Farag and M. Ibrahim \\ Physics Department, Faculty of Science, Ain Shams University, Abbasia \\ 11566, Cairo, Egypt
}

An analytical design of a dielectric multilayer reflector for the visible and near IR spectral range is suggested. It consists of a certain number of stacks arranged (spectrally) beside each other. The left band spectral edge of one stack coincides with the right band spectral edge of its neighboring one. The number of stacks depends on the spectral range needed. Each stack is composed of a specific number of periods which depends on the reflectance desired. Each period contains two quarter wave layers of high and low refractive indices materials. Stacks of quarter wave layers are of considerable interest because of optical monitoring during deposition. Two, three, four and five stacks reflectors are designed taking into consideration the material refractive index dispersion. High expensive computer programs or numerical optimizations are not needed.

\section{Introduction}

Optical coating is used to obtain material surfaces with certain desired optical properties such as reflectance, absorbance and transmittance. High reflectance is needed in Bragg reflectors, dielectric mirrors and optical routers. Design of a multilayer thin-film coating can be approached by an analytical, a numerical method, or the commercial thin-film software design programs. Analytical design methods are valuable not only for coating design but also for understanding coatings behavior [1-3].

Broad-band high-reflectance coatings can be classified into total reflectors $(\mathrm{R} \approx 100 \%)$ and partial reflectors $(\mathrm{R} \leq 90 \%)$. Partial reflectors find applications in laser systems, Fabry-Perot and other types of interferometers. There are several types of broad -band reflectors that are based on single opaque metal layer, all-dielectric multilayer or metal-dielectric multilayer systems [4]. All-dielectric reflecting coatings were based on stacks consisting of alternating high and low refractive index materials. However, with the available 
coating materials, it may not be possible to provide a high reflectance over the desired broad spectral region. Stacks of quarter-wave layers are of considerable interest because of optical monitoring during deposition. However, a quarterwave stack has a limited width of the high reflectance zone [4].

There are different techniques to produce coatings having a wide reflectance zone. The first one consists of depositing a number of quarter-wave stacks on top of each other with different reference wavelengths [5]. Two or more quarter-wave stack reflectors with different periods resulting in contiguous high reflectance bands [5]. In a stack of an alternating high and low refractive index materials, adjacent layers $i$ and $i+1$ were monitored to be quarter-wave layers at wavelengths $\lambda_{\mathrm{i}}$ and $\lambda_{\mathrm{i}+1}$, respectively, such that: $\left(1 / \lambda_{\mathrm{i}}-1 / \lambda_{\mathrm{i}+1}=\right.$ constant $)[6,7]$. The disadvantage of this technique is the occurrence of transmission peaks in the high reflectance zone. A single coupling layer with thickness of one quarter-wave is inserted between the two overlapping stacks to solve this problem $[6,7]$. The broadest region of high reflectance is achieved on the expense of a larger number of layers and larger overall optical thickness of the resulting layer system.

The second technique consists of depositing a stack where the thicknesses of the layers are staggered in some way. Various thickness progressions such as geometric or arithmetic have been tried [8, 9]. The disadvantage of this technique is that the phase of the reflected waves varies rapidly with wavelength. Also there is an increase in the absorption losses. Another approach was based on the use of two or more quarter-wave stack reflector with different periods resulting in contiguous high reflectance bands $[6,7]$. The overlapping of high reflectance bands of different stacks may occur and this result in undesirable reflectance minima.

In this work dielectric multilayer reflectors are designed analytically. It consists of a certain number of stacks arranged spectrally beside each other. The number of stacks depends on the spectral range desired. The left band edge of one stack coincides with the right band edge of its neighboring one. Each stack contains a specific number of periods which depends on the reflectance needed. Each period consists of two layers of different materials: one of them with high refractive index and the other with low refractive index. The material dispersion of the low, high and substrate materials are taken into consideration. Two, three, four and five stack reflectors are designed for visible and near IR spectral range.

\section{Material refractive index dispersion}

Titanium Dioxide $\mathrm{TiO}_{2}$ is used as a high refractive index material of dispersion relation [10]: 


$$
n_{L}^{2}=5.1913+\frac{0.2441 \delta^{2}}{8^{2}-0.0000}
$$

Silicon Dioxide $\mathrm{SiO}_{2}$ is used as a low refractive index material of dispersion relation [10]:

$$
u_{E}^{2}-1+\frac{0.66804 \delta^{2}}{\delta^{2}-0.0026}
$$

The dispersion relation for the substrate material (BK7 Schott) is [11]:

$$
\mathrm{n}_{\mathrm{s}}^{2}=1+\frac{1.00962^{2}}{\lambda^{2}-0.006}
$$

\section{Calculation of spectral characteristics of stacks}

Each layer of a multilayer coating has its own transfer matrix. The overall transfer matrix of the system is the product of the individual transfer matrices, taken in the order in which the light propagates through the multilayer stack. The characteristic matrix of reflector is $[12,13]$ :

$$
M=\left[\begin{array}{ll}
M_{11} & M_{12} \\
M_{21} & M_{22}
\end{array}\right]-\prod_{f=1}^{q} M_{f}
$$

where $\mathrm{q}$ is the number of layers, and the product is taken in reverse order since the matrices of upper layers must be multiplied on the left.

The amplitude reflection and transmission coefficients of the reflector are:

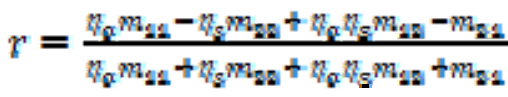

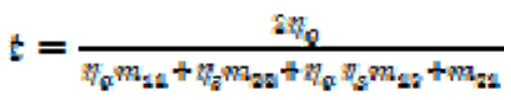

${ } k_{\sigma}=n_{\sigma} \cos \theta_{\sigma}$; for transverse electric polarized light in incidence medium.

$\eta_{o}=n_{\kappa} / \cos \theta_{\kappa} \quad$; for transverse magnetic polarized light in incidence medium.

$\eta_{g}=n_{g} \cos \theta_{g}$; for transverse electric polarized light in the substrate.

$\eta_{a}=n_{a} / \cos \sigma_{z}$; for transverse magnetic polarized light in the substrate.

The reflectance, transmittance and absorbance can be, respectively, written as:

$$
R=r r^{n}=|r|^{2}
$$




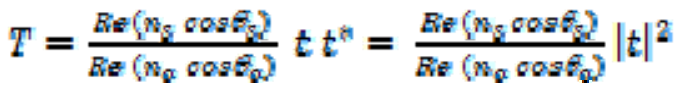

$$
\begin{aligned}
& A=1-R-T
\end{aligned}
$$

where $\mathrm{Re}$ is the real part and * denotes the complex conjugate.

\section{Number of periods for desired reflectance}

The reflectance for $\mathrm{N}$ periods, each is double-layer of quarter-wave thickness, at the central monitoring wavelength, can be written as [14]:

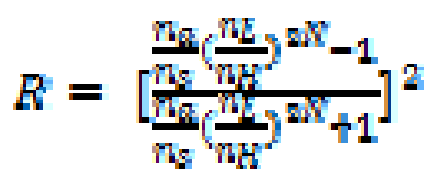

Where $\mathrm{n}_{\mathrm{a}}$ and $\mathrm{n}_{\mathrm{s}}$ are the refractive index of air and substrate respectively.

The number of periods $\mathrm{N}$ can be deduced from equation (10) as:

$$
N=\frac{\ln \left[\frac{\gamma_{g}(1-\sqrt{R})}{\eta_{a}(1+\sqrt{R})}\right]}{2 \ln \left(\frac{n L}{n H}\right)}
$$

\section{Band edges of a dielectric quarter-wave stack}

A reflectance coating can be designed by use of a dielectric quarter-wave stack of alternate high- and low- refractive index materials. The basic building block is (HL) in which $\mathrm{H}$ and $\mathrm{L}$ denote the quarter-wave layer of high- and lowrefractive index materials, respectively. Suppose that the refractive index and the geometrical thickness of the two materials are represented as $\left(n_{H}, t_{H}\right)$ and $\left(\mathrm{n}_{\mathrm{L}}, \mathrm{t}_{\mathrm{L}}\right)$ respectively. The characteristic matrix of the basic block (HL) at an incident angle $\theta_{\mathrm{o}}$ in an incident medium of refractive index $\mathrm{n}_{\mathrm{o}}$ is given by [14]:

$$
\left[\begin{array}{ll}
M_{11} & M_{12} \\
M_{21} & M_{22}
\end{array}\right]=\left[\begin{array}{cc}
\cos \delta_{L t} & t \sin \delta_{L} / \eta_{H} \\
t \eta_{L L} \sin \delta_{H} & \cos \delta_{L}
\end{array}\right]\left[\begin{array}{cc}
\cos \delta_{L} & t \sin \delta_{L} / \eta_{L} \\
t \eta_{L} \sin \delta_{L} & \cos \delta_{L}
\end{array}\right]
$$

where $\delta_{E}$ and $\delta_{L}$ are the optical phase thicknesses of the $\mathrm{H}$ and the $\mathrm{L}$ layers, respectively. The optical phase thickness of each layer can be expressed as:

$$
\delta_{i}={ }_{i}^{2 \pi} \tau_{i} t_{i} \cos z_{z}
$$


where $\mathrm{j}=\mathrm{H}$ or $\mathrm{j}=\mathrm{L}$ and $\theta_{\mathrm{j}}$ is the angle of propagation in the $\mathrm{j}^{\text {th }}$ layer determined by Snell's law: $\mathrm{n}_{\mathrm{o}} \sin \theta_{\mathrm{o}}=\mathrm{n}_{\mathrm{j}} \sin \theta_{\mathrm{j}}$. It should be noted that the optical admittance of the $\mathrm{H}$ or the $\mathrm{L}$ layers, is written as:

$$
\eta_{i}=n_{i} \cos \theta_{i}
$$

for transverse electric polarized light, and by

$$
n_{j}=n_{j} / \cos \theta
$$

for transverse magnetic polarized light. Equations 14 and 15 are for any angle of incidence, but in case of normal incidence there is no difference between the two states of polarizations.

It is known that the boundary condition between the stop and the pass bands in a high reflectance coating can be represented by [13]:

$$
\frac{M_{\mu}+M_{N \mathrm{~N}}}{2}=-1
$$

From the characteristic matrix, this condition can be expressed as:

$$
\cos \delta_{2 L} \cos \delta_{L}-\frac{1}{2}\left(\frac{n_{L}}{\eta_{H}}+\frac{r_{H}}{r_{L}}\right) \sin \delta_{i L} \sin \delta_{L}=-1
$$

Put $\delta_{L} \approx \delta_{L} \approx \delta=\frac{\pi \hat{\delta}_{\alpha}}{2 \lambda}$, and $\eta_{L}=n_{L E} \eta_{L_{L}}=n_{L}$ in case of normal incidence.

Substituting by these values, the left (short) edge for the wavelength range can be written as:

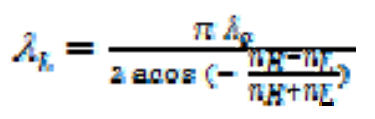

where, $\lambda_{0}$, is the monitoring wavelength. The right (long) edge wavelength, $\lambda_{R}$, is:

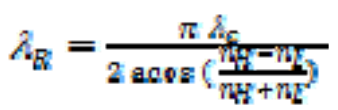

The spectral range of the stack is the difference between the right and left wavelengths.

\section{Steps for analytical designing of a reflector}


1- Specify the desired working wavelength range of the reflector $(400-800 \mathrm{~nm}$ for example).

2- Choose the left (short) edge (for example $\lambda_{\mathrm{L} 1}=400 \mathrm{~nm}$ ) for the wavelength range of the first stack, then deduce its monitoring wavelength, $\lambda_{\mathrm{ol}}$, from the relation:

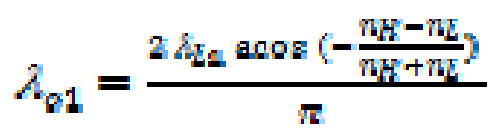

3- Determine the right (long) edge wavelength of the first stack, $\lambda_{\mathrm{R} 1}$, from the relation:

$$
\lambda_{R 1}=\frac{\pi \delta_{\alpha_{4}}}{2 \operatorname{acos}\left(\frac{h_{H}-n_{E}}{n_{H}+n_{L}}\right)}
$$

4- Take the right edge wavelength of the first stack equals to the left edge wavelength of the second stack $\lambda_{B 1}-\lambda_{L 2}$

5- Determine the monitoring wavelength, $\lambda_{02}$, of the second stack from the relation:

$$
\lambda_{\gamma_{2}}=\frac{2 \cdot \delta_{\Sigma_{n}} \operatorname{acos}\left(-\frac{n_{H}-n_{\varepsilon}}{n_{H}+n_{\varepsilon}}\right)}{\pi}
$$

6- Determine the right (long) edge wavelength of the second stack, $\lambda_{\mathrm{R} 2}$, from the relation:

$$
\lambda_{R 2}=\frac{\pi \hat{A}_{\alpha 2}}{2 \operatorname{acos}\left(\frac{n_{H}-n_{L}}{n+n_{L}}\right)}
$$

7- Take the right edge wavelength of the second stack equals to the left edge wavelength of the third stack $Q_{1} R 2=Q_{1} L 3$

8- Repeat these steps till the final edge of the desired wavelength range is reached.

9- The number of periods for each stack (at certain monitoring wavelength) increases as we proceed from short wavelength region (high dispersion) towards long wavelength region (low dispersion), i.e., the number of periods for each stack equals the stack number.

\section{Results and Discussion}


Two, three, four and five stack reflectors are designed following the steps which are explained in section 6. Table (1) shows the thicknesses and monitoring wavelengths for the design of two stack reflector. This reflector in the form: $\mathrm{a}\left(\mathrm{H}_{1} \mathrm{~L}_{1}\right)^{1}\left(\mathrm{H}_{2} \mathrm{~L}_{2}\right)^{2} \mathrm{~g}$, where $\mathrm{a}$ is the air and $\mathrm{g}$ is the substrate glass medium. Fig. (1) shows the reflectance of two stack reflectors. The average reflectance of this reflector is about $90 \%$, starts at $370 \mathrm{~nm}$ and ends at $615 \mathrm{~nm}$ with spectral width of about $245 \mathrm{~nm}$.

Table (1): Thicknesses and monitoring wavelengths of two stack reflector

\begin{tabular}{|l|l|l|l|}
\hline Stack number & $\begin{array}{l}\text { High index } \\
\text { thickness }(\mathrm{nm})\end{array}$ & $\begin{array}{l}\text { Low index } \\
\text { thickness }(\mathrm{nm})\end{array}$ & $\begin{array}{l}\text { Monitoring } \\
\text { Wavelength }(\mathrm{nm})\end{array}$ \\
\hline 1 & 38.067 & 68.82 & 355 \\
\hline 2 & 55.332 & 100.032 & 516 \\
\hline
\end{tabular}

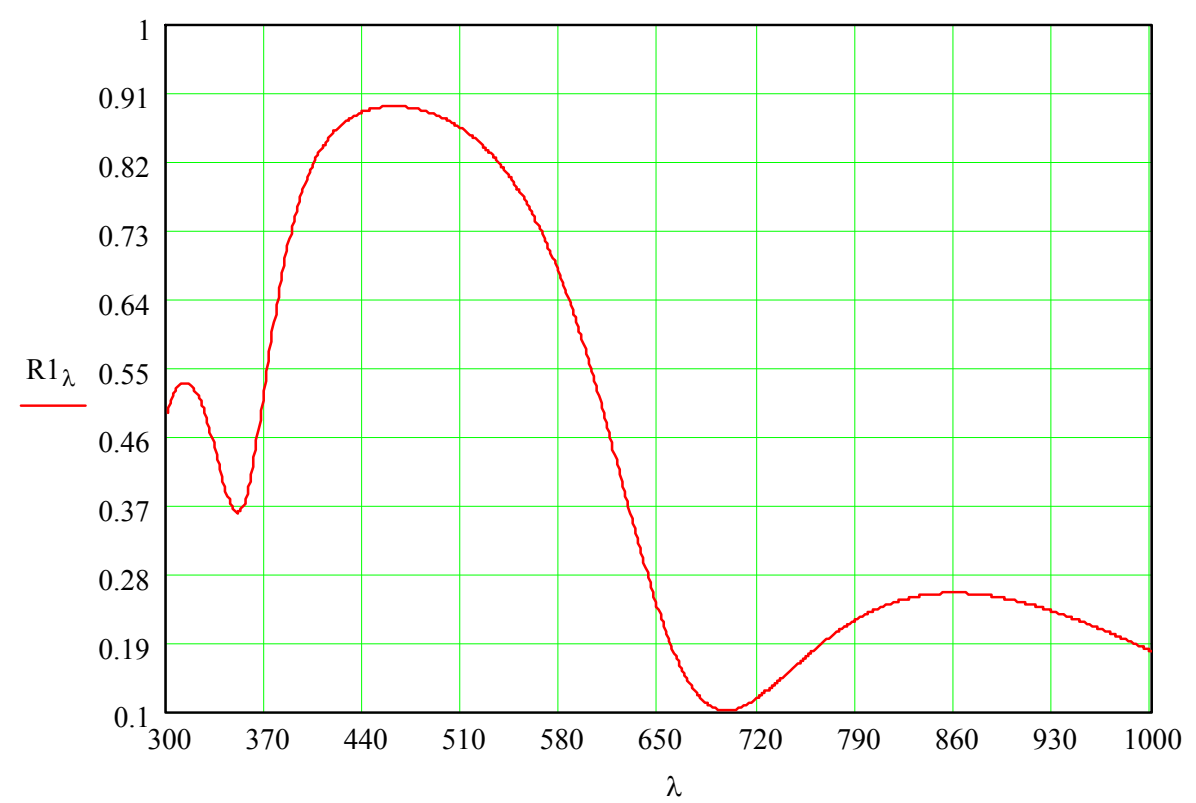

Fig. (1): Reflectance of two stack reflector with design: $a\left(H_{1} L_{1}\right)^{1}\left(H_{2} L_{2}\right)^{2} g$

Table 2 shows the thicknesses and monitoring wavelengths of the three stack reflector. This design has three stacks in the form: $a\left(\mathrm{H}_{1} \mathrm{~L}_{1}\right)^{1}\left(\mathrm{H}_{2} \mathrm{~L}_{2}\right)^{2}$ $\left(\mathrm{H}_{3} \mathrm{~L}_{3}\right)^{3} \mathrm{~g}$. Fig. (2) shows the reflectance of the three stack reflector across the desired spectral range. The average reflectance is about $90 \%$ and the spectral range of the reflector extends from $350 \mathrm{~nm}$ to $760 \mathrm{~nm}$ with a spectral width of about $410 \mathrm{~nm}$.

Table (2): Thicknesses and monitoring wavelengths of three stack reflector 


\begin{tabular}{|l|l|l|l|}
\hline Stack number & $\begin{array}{l}\text { High index } \\
\text { thickness }(\mathrm{nm})\end{array}$ & $\begin{array}{l}\text { Low index } \\
\text { thickness }(\mathrm{nm})\end{array}$ & $\begin{array}{l}\text { Monitoring } \\
\text { Wavelength }(\mathrm{nm})\end{array}$ \\
\hline 1 & 38.067 & 68.82 & 355 \\
\hline 2 & 49.541 & 89.563 & 462 \\
\hline 3 & 72.06 & 130.274 & 672 \\
\hline
\end{tabular}

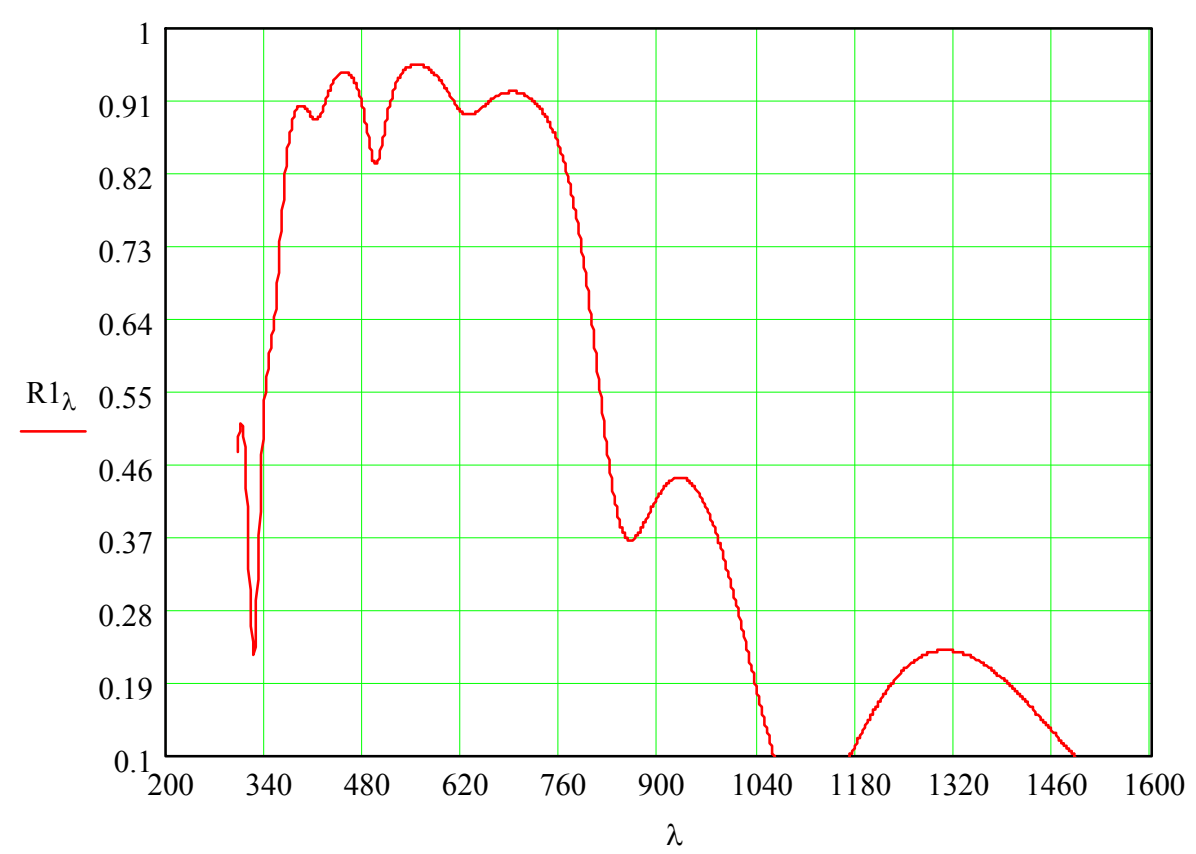

Fig. (2): Reflectance of three stack reflector with design: $a\left(H_{1} L_{1}\right)^{1}\left(H_{2} L_{2}\right)^{2}\left(H_{3} L_{3}\right)^{3} g$.

Table 3 shows the thicknesses and monitoring wavelengths of the four stack reflector. This design has four stacks with the form a $\left(\mathrm{H}_{1} \mathrm{~L}_{1}\right)^{1}\left(\mathrm{H}_{2} \mathrm{~L}_{2}\right)^{2}$ $\left(\mathrm{H}_{3} \mathrm{~L}_{3}\right)^{3}\left(\mathrm{H}_{4} \mathrm{~L}_{4}\right)^{4} \mathrm{~g}$.

Table (3): Thicknesses and monitoring wavelengths of four stack reflector

\begin{tabular}{|l|l|l|l|}
\hline Stack number & $\begin{array}{l}\text { High index } \\
\text { thickness }(\mathrm{nm})\end{array}$ & $\begin{array}{l}\text { Low index } \\
\text { thickness }(\mathrm{nm})\end{array}$ & $\begin{array}{l}\text { Monitoring } \\
\text { Wavelength }(\mathrm{nm})\end{array}$ \\
\hline 1 & 38.067 & 68.82 & 355 \\
\hline 2 & 48.898 & 88.40 & 456 \\
\hline 3 & 71.095 & 128.529 & 663 \\
\hline 4 & 103.372 & 186.831 & 964 \\
\hline
\end{tabular}


Figure (3) shows the reflectance of this reflector which has average reflectance of about $90 \%$. The spectral range extends from $480 \mathrm{~nm}$ to $1200 \mathrm{~nm}$ with a spectral width of about $720 \mathrm{~nm}$.

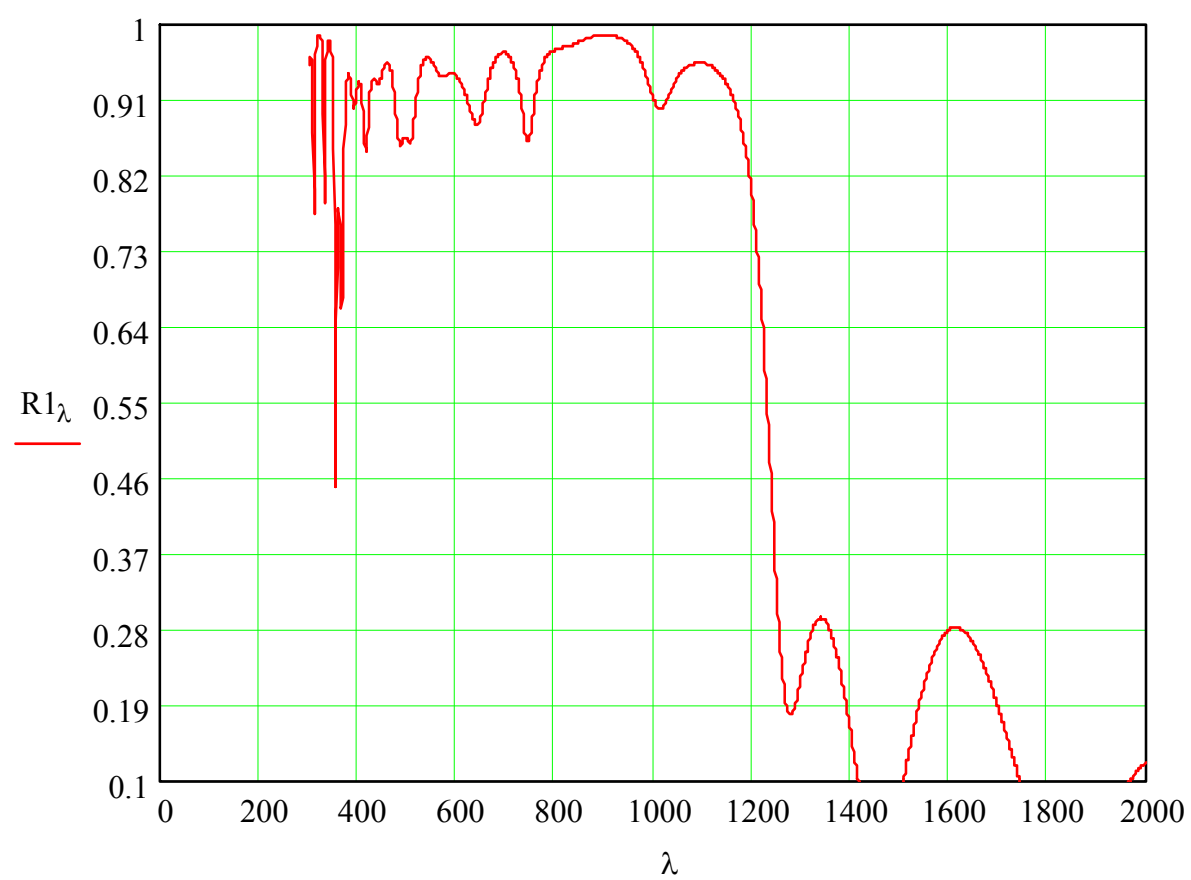

Fig. (3): Reflectance of four stack reflector with design: $a\left(\mathrm{H}_{1} \mathrm{~L}_{1}\right)^{1}\left(\mathrm{H}_{2} \mathrm{~L}_{2}\right)^{2}$ $\left(\mathrm{H}_{3} \mathrm{~L}_{3}\right)^{3}\left(\mathrm{H}_{4} \mathrm{~L}_{4}\right)^{4} \mathrm{~g}$.

Table 4 shows the thicknesses and monitoring wavelengths of the five stack reflector. The reflector has the form a $\left(\mathrm{H}_{1} \mathrm{~L}_{1}\right)^{1}\left(\mathrm{H}_{2} \mathrm{~L}_{2}\right)^{2}\left(\mathrm{H}_{3} \mathrm{~L}_{3}\right)^{3}\left(\mathrm{H}_{4} \mathrm{~L}_{4}\right)^{4}$ $\left(\mathrm{H}_{4} \mathrm{~L}_{4}\right)^{4}$ g. Fig. (4) shows the reflectance of the five stack reflector which has an average reflectance of about $93 \%$ and the spectral range extends from $500 \mathrm{~nm}$ to $1800 \mathrm{~nm}$ with a spectral width of about $1300 \mathrm{~nm}$.

Table (4): Thicknesses and monitoring wavelengths of the five stack reflector

\begin{tabular}{|l|l|l|l|}
\hline Stack number & $\begin{array}{l}\text { High index } \\
\text { thickness }(\mathrm{nm})\end{array}$ & $\begin{array}{l}\text { Low index } \\
\text { thickness }(\mathrm{nm})\end{array}$ & $\begin{array}{l}\text { Monitoring } \\
\text { Wavelength (nm) }\end{array}$ \\
\hline 1 & 38.067 & 68.82 & 355 \\
\hline 2 & 48.254 & 87.237 & 450 \\
\hline 3 & 70.13 & 126.784 & 654 \\
\hline 4 & 101.978 & 184.361 & 951 \\
\hline 5 & 148.409 & 268.302 & 1384 \\
\hline
\end{tabular}




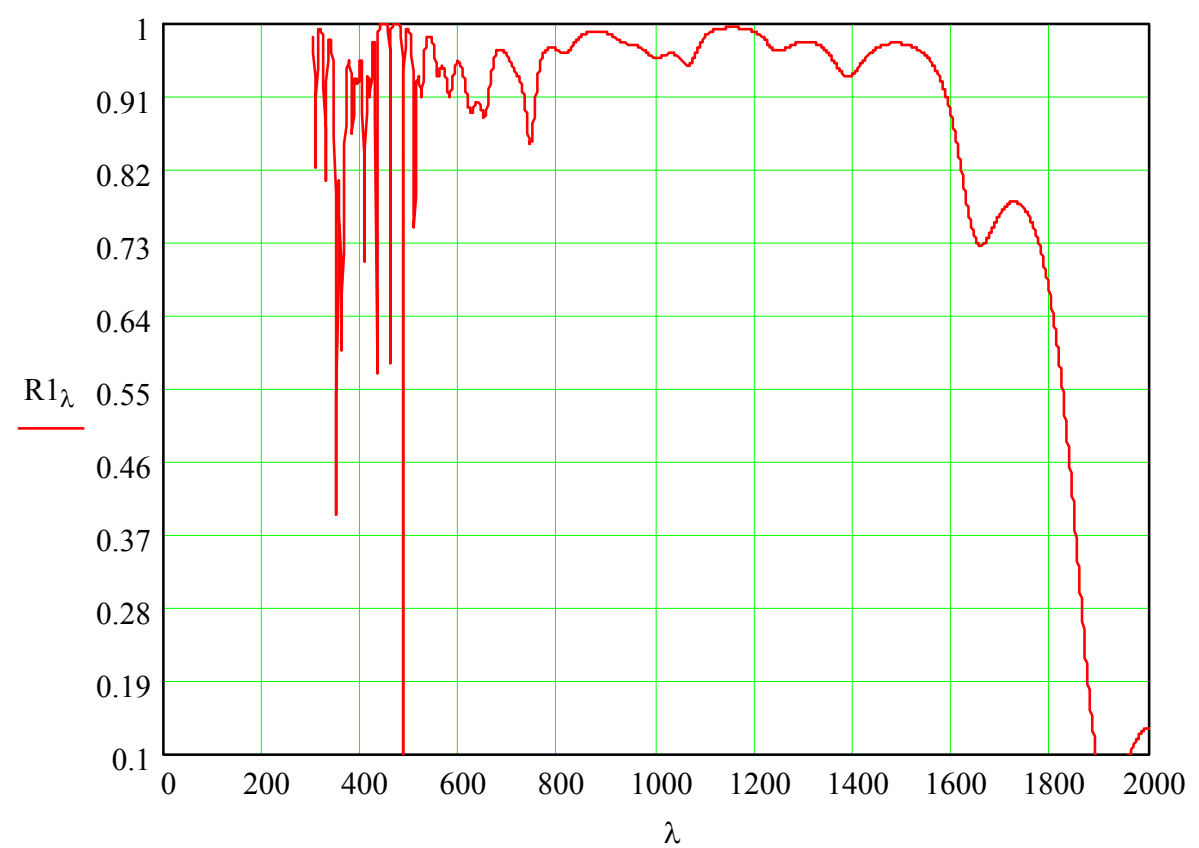

Fig. (4): Reflectance of the five stack reflector with design: $a\left(H_{1} L_{1}\right)^{1}\left(H_{2} L_{2}\right)^{2}$ $\left(\mathrm{H}_{3} \mathrm{~L}_{3}\right)^{3}\left(\mathrm{H}_{4} \mathrm{~L}_{4}\right)^{4}\left(\mathrm{H}_{5} \mathrm{~L}_{5}\right)^{4} \mathrm{~g}$.

From the previous tables and figures, it can be shown that as the number of stacks increases the spectral width of the reflector increases with a shift of the short wavelength edge towards the longer wavelengths. The reflectance of the different reflectors is nearly constants across the desired wavelengths range.

\section{Conclusion}

A simple analytical procedure is presented to design a wideband reflector for the visible and near infrared spectral range. The reflectance of the reflector is selected by the number of periods in the stack. A number of contiguous stacks are arranged, spectrally, side by side depending on the spectral range needed. Computer optimization or high expensive design programs are not needed. Stacks of quarter wave layers are used in the designs to facilitate the optical monitoring during materials deposition. 


\section{References}

1. R. R. Willey, "Practical Design and Production of Optical Thin Films", Marcel Dekker, New York, p. 85 (2002).

2. R. R. Willey, Field Guide to Optical Thin Films: SPIE Field Guides volume FG07, SPIE Press, Bellingham, p. 84 (2006).

3. H. K. Pulker, Coating on Glass, Elsevier, Amsterdam, p. 429 (1999).

4. A. Thelen, Design of Optical Interference Coating, McGraw-Hill, New York, p. 87 (1989).

5. S. Penselin and A. Stendel, Z. Phys. 142, 21(1955).

6. D. L. Perry, Proc. IEEE, 53, 76 (1965).

7. A. F. Turner and P. W. Baumeister, Appl. Opt., 5, 69 (1966).

8. O. S. Heavens and H. M. Liddell, App. Opt. 5, 373 (1966).

9. F. A. Korolev, A. Y. Klemnteva, T. F. Meshcheryakova and I. A. Ramazina, Opt. Spectros. (USSR) 28, 416 (1970).

10. M. J. Weber, Handbook of Optical Materials, CRC Press, London, p. 100 (2003).

11. www.us.schott.com/advanced optics/.../index.htm

12. H. A. Macleod, Thin Film Optical Filters, Macmillan, New York, p. 158 (1986).

13. P. Yeh, Optical Waves in Layered Media, Wiley, New York, p. 102 (1988).

14. F. L. Pedrotti and L. S. Pedrotti, Introduction to Optics, Prentice- Hall International, Inc., London, p. 402 (1987). 